\title{
Micropower Impulse Radar Technology and Applications
}

\author{
J. Mast, S. Azevedo, W. Haddad, \\ L. Ng, G. Burnett
}

April 15, 1998

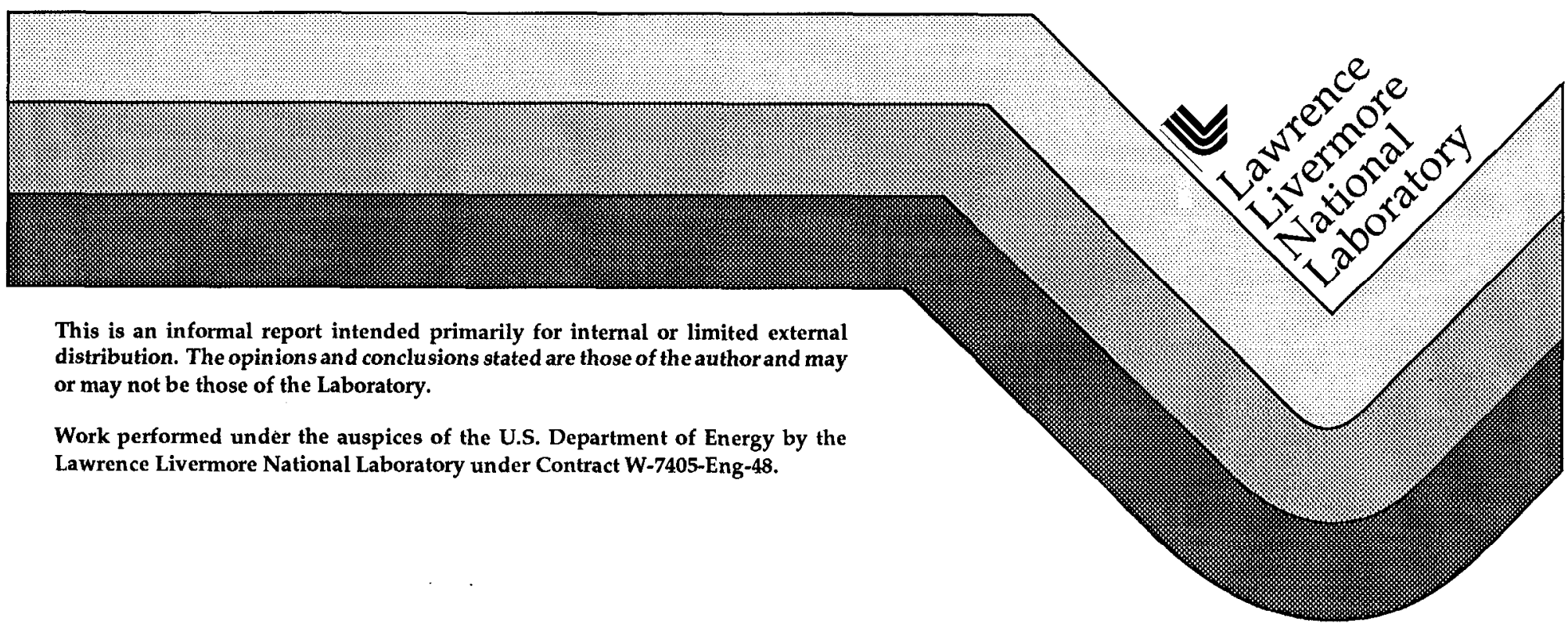




\section{DISCLAIMER}

This document was prepared as an account of work sponsored by an agency of the United States Government. Neither the United States Government nor the University of California nor any of their employees, makes any warranty, express or implied, or assumes any legal liability or responsibility for the accuracy, completeness, or usefulness of any information, apparatus, product, or process disclosed, or represents that its use would not infringe privately owned rights. Reference herein to any specific commercial product, process, or service by trade name, trademark, manufacturer, or otherwise, does not necessarily constitute or imply its endorsement, recommendation, or favoring by the United States Government or the University of California. The views and opinions of authors expressed herein do not necessarily state or reflect those of the United States Government or the University of California, and shall not be used for advertising or product endorsement purposes.

This report has been reproduced

directly from the best available copy.

Available to DOE and DOE contractors from the Office of Scientific and Technical Information

P.O. Box 62, Oak Ridge, TN 37831

Prices available from (423) 576-8401

Available to the public from the

National Technical Information Service

U.S. Department of Commerce

5285 Port Royal Rd.,

Springfield, VA 22161 


\title{
Micropower Impulse Radar Technology and Applications
}

\author{
J. Mast, S. Azevedo, W. Haddad, L. Ng, G. Burnett
}

\section{Introduction}

The LLNL-developed Micropower Impulse Radar (MIR) technology has quickly gone from laboratory concept to embedded circuitry in numerous government and commercial systems in the last few years[1]. The main ideas behind MIR, invented by T. McEwan in the Laser Program, are the generation and detection systems for extremely low-power ultra-wideband pulses in the gigaHertz regime using low-cost components. These ideas, coupled with new antenna systems, timing and radio-frequency (RF) circuitry, computer interfaces, and signal processing, have provided the catalyst for a new generation of compact radar systems. Over the past several years we have concentrated on a number of applications of MIR which address a number of remote-sensing applications relevant to ennerging prograns in defense, transportation, medical, and envirommental research. Some of the past commercial successes have been widely publicized [2] and are only now starting to become available for market. Over 30 patents have been filed and over 15 licenses have been signed on various aspects of the MIR technology. In addition, higher performance systems are under development for specific laboratory programs and government reimbursables.

The MIR is an ultra-wideband, range-gated radar system that provides the enabling hardware technology used in the research areas mentioned above. It has numerous performance parameters that can be selected by careful design to fit the requirements. We have improved the baseline, short-range, MIR system to demonstrate its effectiveness. The radar operates over the band from approximately 1 to $4 \mathrm{GHz}$ with pulse repetition frequencies up to $10 \mathrm{MHz}$. It provides a potential range resolution of $1 \mathrm{~cm}$ at ranges of greater than $20 \mathrm{~m}$. We have developed a suite of algorithms for using MIR for image formation. These algorithms currently support synthetic aperture and multistatic array geometries. This baseline MIR radar imaging system has been used for several programmatic applications.

\section{Background}

MIR is an entirely new sensor concept that is based on high-speed pulsed electronics developed by Engineering and the Laser Program. The radars exhibit a combination of interesting properties including, wide band operation, extremely low power consumption, extremely small size and low cost, array configurability, noise-encoded pulse generation, and other features. Two MIR sensors form the foundation for this work - the motion sensor and the distance sensor. These sensors form a foundation for the development of other more elaborate systems when multiple sensors are coupled with advanced signal processing techniques.

\section{Motion Sensors}

The motion sensor provides a small low-cost sensor that detects motion or vibration. We have demonstrated that the sensor can pick up the motion of vocal cords and other objects that vibrate at a range of frequencies. Micropower radars, designed for the purposes of speech coding, have been shown to dramatically increase the accuracy of speech recognition, of speaker verification, of near instantaneous phrase translation, and of speech synthesis. This sensor has the unique characteristic that it can be used both directly coupled to and offset from an object. This capability could prove extremely useful in machine or structural monitoring applications where vibration analysis is used to determine failure modes. MIR could prove to be a flexible new sensor for this application providing additional information complementing acoustic accelerometers, laser interferometers, and other traditional vibration monitoring devices.

\section{Distance Sensors}

The MIR Rangefinder is the most suphisticaled of the dozens of MIR prototypes; it is the basis of all imaging applications and of many reimbursable projects. The device has been modularized allowing a tremendous amount of flexibility for adaptation to a wide variety of applications. The radar gencrates an equivalent-time A-scan with a typical range sweep of $10 \mathrm{~cm}$ to $3 \mathrm{~m}$ and an incremental range resolution, as limited by noise, of 0.3 min. The modular system accepts a standard 50 Ohm matched UWB antenna and operates with a bandwidth from approximately 1 to 4 GHz. Increasing the band width of the sensor to include frequencies up to $10 \mathrm{GHz}$ is technically feasible and would broaden the application areas for which this sensor can apply. 


\section{Imaging Systems}

The basic MIR Rangefinder above provides the perfect vehicle for ultra-wideband imaging applications. These radars are coupled to a portable computer to form complete radar systems with either synthetic aperture or real-array imaging. Radar return signals are digitized and stored in a portable laptop computer. Reconstruction of cross-sectional images from B-scan or waterfall type data is performed by diffraction tomography software on the lap-top[5]. Images of the scene are displayed directly on the screen within 10 seconds (in 2D). We have demonstrated the use of this radar package for integration into an imaging array that is small, lightweight, low power, and inexpensive relative to existing radars.

\section{Major Accomplishments and Results}

The focus of our effort has been in the aforementioned application areas. We have developed array imaging systems for road-bed and bridge-deck inspection, landmine and buried ordnance detection, and through-wall personnel detection. These systems have employed MIR rangefinder sensors integrated into multi-element arrays. Extensive antenna design and improved sensor acquisition rates have been incorporated into these systems. We have explored medical applications that involve using sensitive MIR motion sensors to measure internal body motion. For example, an MIR heart sensor noninvasively measures motion of the heart muscle and appears to provide signals of some physiological significance. We also detect small breathing motions that originate under several layers of concrete. Non-acoustical devices based on MIR have bcen shown to enhance information content of standard acoustical measurements. For example, motion sensors similar to the heart monitor can measure the motion of vocal cords, tongue, and lips providing additional information to aid in speech recognition.

Over the course of this research project we have made significant technical progress. Initial testing of an array of microradar transmit/receive modules has been successful in demonstrating mine detection and real-time personnel motion behind walls. We have successfully engineered PRF improvements from $40 \mathrm{~Hz}$ to $>5 \mathrm{kHz}$ enabling high acquisition rates from MIR arrays. In addition to the prototype array, we have built and demonstrated a prototype rubble rescue radar system for locating people beneath rubble. We have achieved simple phonetic speech synthesis utilizing the MIR spccch radar along with successful acquisition of simultaneous glottal radar, EGG, audio, and airflow data. We have established collaborations with UC Berkeley to integrate the radar into a full scale Hidden Markov Model recognizer. For medical applications of MIR we have successfully performed dielectric measurements of organs and tissues of a live pig in collaboration with Watter Reed Army Institute of Research (WRAIR). These data provide an invaluable database for future experiments and analysis of MIR sensors for medical applications. Additionally we have developed a MIR heart monitor that is significantly smaller than previous prototypes. Collaborations have been established with WRAIR to do further research with this prototype in conjunction with the development of a human heart phantom to gain better understanding of the heart monitoring sensors.

\section{MIR Sensors}

In an effort to produce functional radar modules that can have many features to aid system development, we have designed and developed a family of MIR boards, antennas, interconnections and software with standard interfaces that "plug and play" together. MIR modular components fall into four general categories of software, computer interfaces, timing and baseband processing circuits, and high-speed front ends. The interconnections between the categories are based on industry standard hardware or sof ware components (for example, SMA or audio connectors, TTL voltages, and $50 \mathrm{ohm}$ terminating resistances). The focus of our effort has been on the timing and high-speed circuitry, where much of the family has alrcady becn implcmented. Incorporated into these modules are many of the anticipated enhancements needed to develop future MIR hardware systems. For this reason, the modular MIR boards are generally larger than the first generation of boards, but their layout is such that subcircuits can be easily reconfigured to build custom boards, or potentially application-specific integrated circuits (ASIC's), for particular applications. Both government and commercial projects are better served by the modular approach, because prototyping of radar systems becomes a simpler task and direct characterization of the individual components is more straight forward than with previous MIR systems.

Significant progress has been made in generating radar components that produce consistent and repeatable responses under most conditions. In contrast to earlier MIR prototypes, the current surface-mount board designs are very robust to shock, interference, and temperature changes. For example, a constant-fraction discriminator (CFD) has been added to the Dipstick and Rangefinder systems that automatically adjusts a threshold detector to a temperature-compensated 
reference voltage. Then distance or range is measured by pulse-width modulation of the time between threshold crossings of MIR impulses. For a 100-ps-wide pulse, the leading edge rise-time corresponds to about $1.5 \mathrm{~cm}$ in range. To hold range errors below $1 \mathrm{~mm}$, we need a threshold detection accuracy of better than $6 \%$, regardless of pulse amplitude. Compensation for voltage and thermal changes (about $1 \%$ fluctuation over outdoor temperatures) is automatic and flexible enough to operate under harsh conditions.

1. MIR Motion Sensor. This is an enhanced version of the single-board motion sensor that can be easily reconfigured to match a specific need. Like the original, it is range-gated, low power (multi-year battery life), low cost, channel-less (multiple MIR units can operate in close proximity without RF interference), and nearly impossible to detect. A photograph of the board with simple quarter-wave antennas is shown in Fig. 2. Only motion-modulated signals or changes from a baseline measurement are detected, thereby eliminating false triggers from stationary room "clutter." The motion pass-band can be changed by modifying the on-board filter components to match the application. An independent laboratory has verified that the MIR motion sensor can satisfy FCC Part 15 regulations. Applications are in security and energy control systems, industrial safety, robotics, vibration sensing, and speech processing.

2. MIR Electronic Dipstick. This is a two-board low-cost time-domain reflectometer (TDR) system that was designed to detect the height of fluid in a reservoir or container by measuring the pulse-echo time of an MIR pulse launched along a transmission line -- a simple wire. The two modular boards are the quartz time base and TDR signal processor described in Fig. 1. Measurement of the fluid height is typically resolved to $0.1 \%$ of maximum range. There are many applications of the system in measuring fluid and material levels in industrial containers (tanks, vats, silos), hazardous materials, down-hole water levels, automotive tank monitoring, and in providing automatic fill control.

3. MIR Rangefinder. This is a five-board complete impulse radar transceiver system with swept range-gate and ultrawideband antennas. A photograph of the full modular Rangefinder is shown in Fig. 3. The five boards used are the quartz time base, Rangefinder signal processor, 60-ps impulse receiver, and two transmitter boards (60-ps impulse and $6.5-\mathrm{MHz}$ pulsed oscillator boards). The receiver works equally well with both impulse and pulsed-oscillating transmitters. Waveform outputs of the two transmitters are shown in Fig. 4. It generates an equivalent-time A-scan (echo amplitude vs. range, similar to a WW-II radar) with a typical echo pattern in the returned signal.

\section{Imaging Systems}

Throughout the course of this research we have developed and improved MIR sensors embedded in imaging systems for both detection of landmines and nondestructively evaluating civil structures such as bridge decks. Additionally we

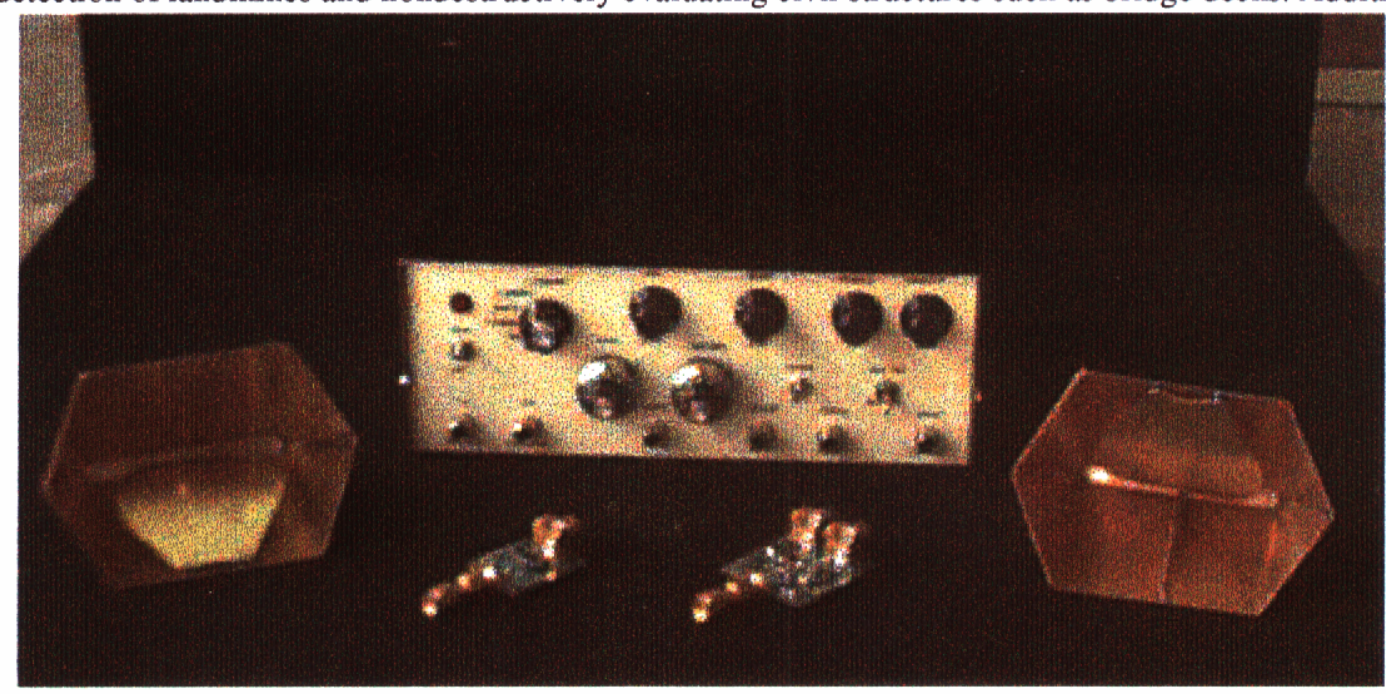

Figure 1 The modular MIR system consisting of a transmitter module, receiver module, two antennas, and the timing and control unit.

have developed synthetic aperture imaging methods and signal processing techniques to generate images from the data acquired using an MIR based radar and investigated potential applications of these systems. 


\section{Road-bed and bridge-deck inspection}

Delamination of concrete structures due to corrosion of rebar layers is a major problem in our-national transportation infrastructure. For structural engineers, the most important features to be detected in concrete bridges are the horizontal (flat) delaminations of concrete layers due to residual corrosion effects in the rebar layers. Using MIR systems combined with signal processing techniques, we have demonstrated the capability to image the delaminations below asphalt and concrete layers as an alternative to coring or other destructive methods (see Figure 2). These delaminations or cracks are either air- or water-filled. The desired inspection performance level is to detect delaminations down to one-millimeter thickness and greater than $10 \mathrm{~cm}$ in extent. This specification along with knowledge of the materials involved, allow modeling of the radar cross section (RCS) of these cracks and hence the acceptable signal-to-noise tolerances for detection.

We have been contracted by the Federal Highway Administration to construct a trailer-mounted imaging radar array that can inspect the roadway for damage at near-highway speeds. Work is ongoing to determine the correlation between radar image analysis and structural integrity and features of the bridge deck.

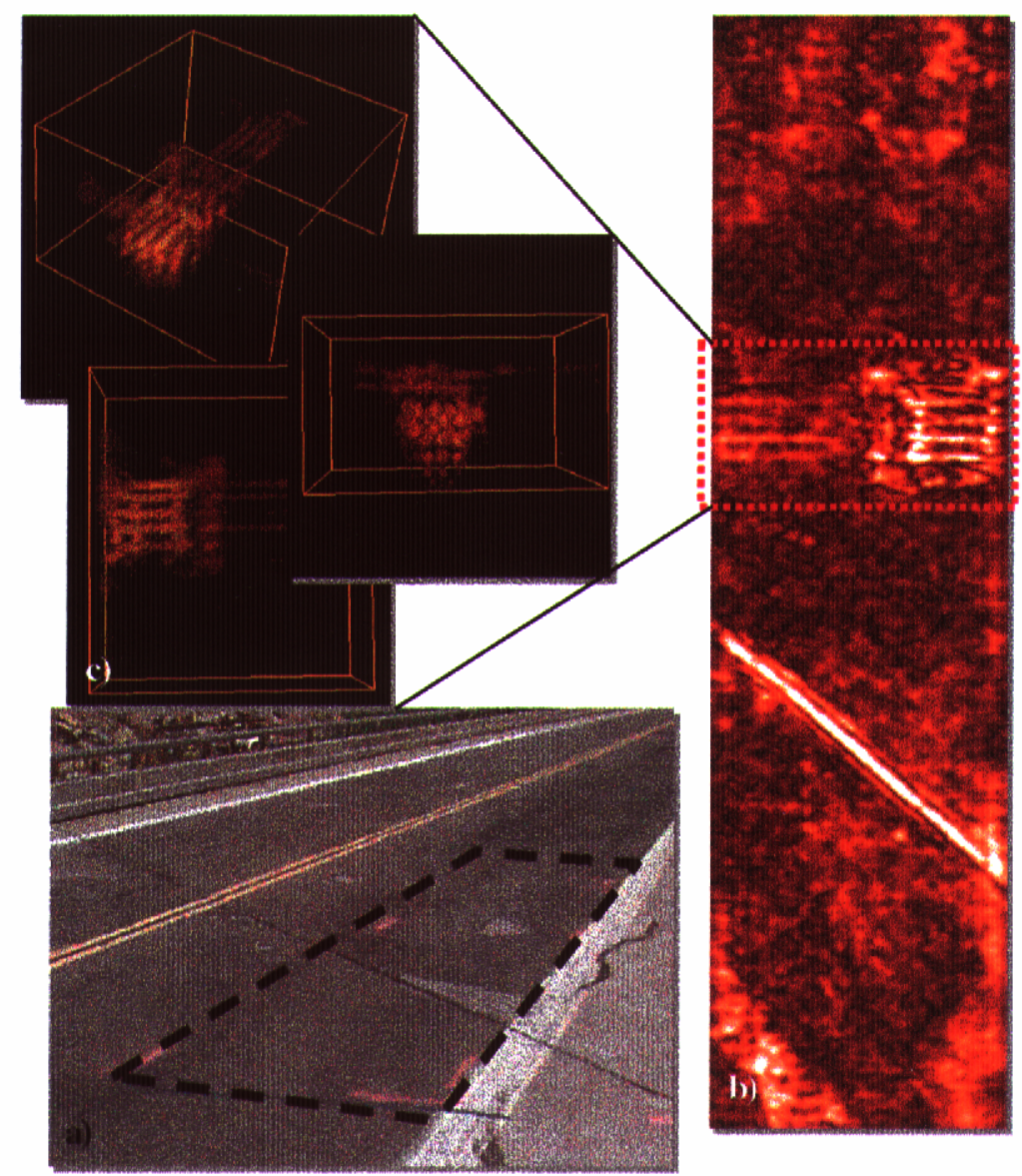

Figure 2 Demonstration of MIR imaging system used for nondestructive evaluation of a bridge deck. (a) region scanned using MIR system, (b) image slice at depth of 15 $\mathrm{cm}$, (c) 3D rendered views of a patched region of the bridge deck visualizing substructure damage. 


\section{Landmine and buried ordnance detection}

With 100 million mines in the world, and more being placed daily, detection and removal of them in an effective reliable way is a major problem both for military and humanitarian objectives. Non-metallic mines pose a particular problem because standard metal detectors are incapable of finding them. The MIR is well suited for both hand-held and vehicle-mounted situations because of its lightweight and low power consumption. Image reconstruction is necessary to ascertain the shape and size of the buried object to distinguish it from ground clutter[3,4]. Buried
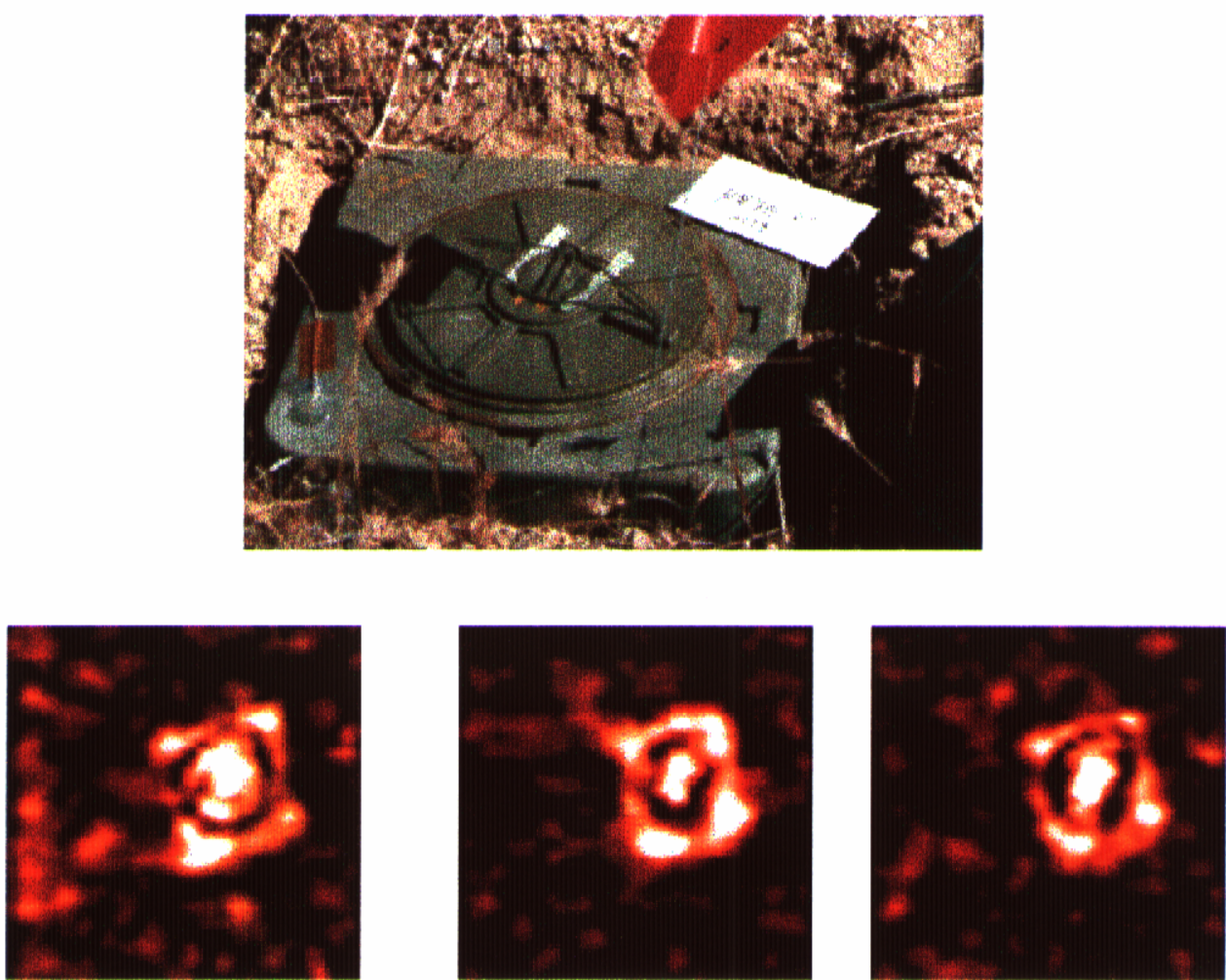

Figure 3 Results of 3D MIR imaging of a buried anti-tank mine at the Nevada Test Site. Images are at depths of 4,5 , and $6 \mathrm{~cm}$ (left to right).

unexploded ordnance presents a similar hazard to civilian rehabitation of an area after war, and MIR imaging could potentially address this problem also, though the specifications are slightly different.

We performed a preliminary evaluation of MIR based mine detection systems on a minefield containing anti-tank mines located at the Nevada Test Site. Using an MIR rangefinder, we scanned several of the buried mines located in the field and generated images that are shown in Figure 3. The resolution of the images is approximately $2 \mathrm{~cm}$ showing structural features of the landmine. Anti-tank mines are large and usually made of metal making them easier to find than anti-personnel mines that are smaller (4-6 $\mathrm{cm}$ in size) and made of plastic. To aid in designing future MIR mine detection systems we performed fundamental imaging experiments to determine the appropriate radar frequency band

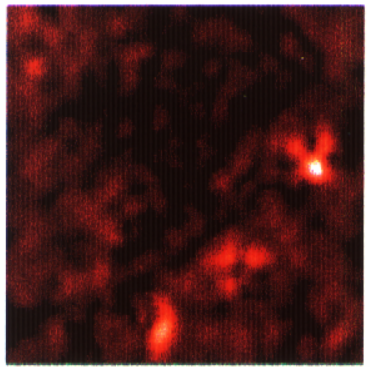

$2.5-5 \mathrm{GHz}$

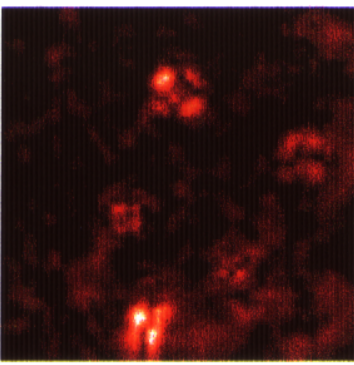

$5-7.5 \mathrm{GHz}$

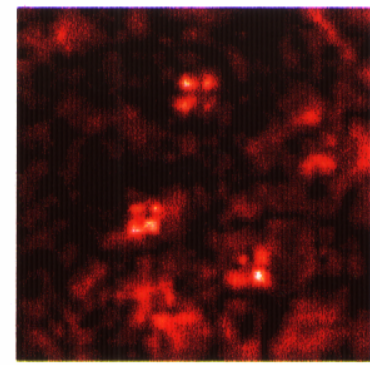

$7.5-10 \mathrm{GHz}$

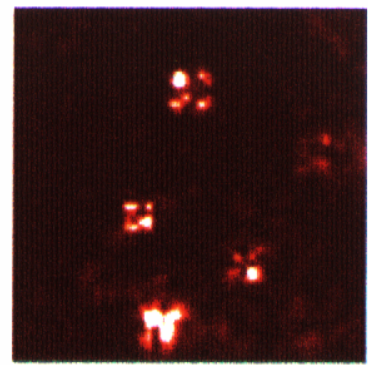

2 looks

Figure 4 Anti-personnel mines imaged in sand using several frequency bands from 2.5 to $10 \mathrm{GHz}$. The image on the right was created using a multi-look imaging technique which combines the results from the two higher frequency bands. 
to use for locating anti-personnel mines. Using an HP network analyzer and a pair of cross-polarized horn antennas we recorded synthetic aperture data from $2.5 \mathrm{GHz}$ to $10 \mathrm{GHz}$ from several anti-personnel mines buried in sand. Images formed from different subbands are shown in Figure 4 and show a considerable improvement in visibility of the mines for the frequency bands above $5 \mathrm{GHz}$. This experiment was only performed for a single soil type and the results will vary depending upon soil conditions. However, the results demonstrate the importance of developing MIR systems covering a higher frequency range than the current 1 to $5 \mathrm{GHz}$.

\section{Medical Sensors}

Central to any fundamental scientific study or technological endeavor is basic physical information about the materials and radiation that are involved with the project, and the interaction between the two. In general, data characterizing material properties is required for numerical modeling and simulation, hardware design, the design and construction of physical models and laboratory experiments, theoretical calculations and estimates, and an overall understanding of how things work. The data must be relatively complete and must be available to the researchers in a usable form. In the area of biomedical applications of microwaves, the materials of interest are the tissues of the body and the radiation being considered is electromagnetic radiation at frequencies from a few kilohertz to a few hundred megahertz. The specific material property of interest is the frequency dependent dielectric constant, more properly called the complex dielectric function, of various body tissues. Scientific research in the area of biomedical applications of microwave devices and related topics dates back to the beginning of this century. Despite considerable activity in this area of research, a relatively complete and consistent data set encompassing both a wide variety of tissue types as well as a wide frequency range is not readily available.

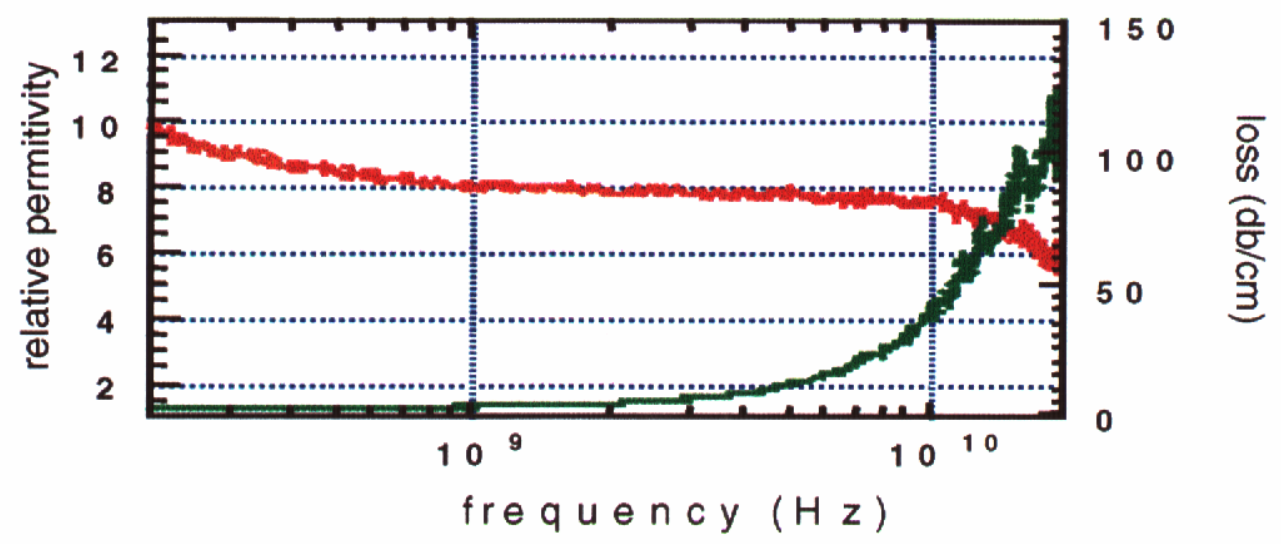

Figure 5 Dielectric measurements (permittivity $=$ red, loss $=$ green) taken from a live pig heart using an HP8720-C nework analyzer with an HP85070-B dielectric probe.

The complex dielectric function of a series of body tissues and fluids were recorded in-vivo and after excision from swine. A total of 26 tissue samples and 5 fluid samples with some additional variations were measured using an HP8720-C network analyzer with an HP85070-B dielectric probe. A total of 801 data points were taken for each sample over the frequency range of $200 \mathrm{MHz}$ to $20 \mathrm{GHz}$. A sample of this data taken from a live pig heart is shown in Figure 5. Each measurement was repeated a minimum of three times in immediate succession on the same region of each sample to ensure consistency and aid in error analysis.

\section{Rubble Rescue Radar (RRR)}

Each year, innocent human lives are lost in collapsed structures as a result of both natural and man-made disasters. We have developed a prototype device pictured in Figure 6, called the Rubble Rescue Radar (RRR) as a aid to workers trying to locate trapped victims in urban search and rescue operations. The RRR is a motion sensor MIR, and is capable of detecting human breathing motions through reinforced concrete. It is lightweight, and designed to be handled by a single operator for localized searches in areas where trapped victims are expected. Tests of the first prototype device were conducted on site at LLNL using a mock rubble pile consisting of a reinforced concrete pipe with two concrete floor slabs placed against one side, and random concrete and asphalt debris piled against the other. This arrangement provides safe and easy access for instruments and/or human subjects. Breathing signals of a human subject were recorded with the RRR through one floor slab plus the wall of the pipe, two slabs plus the wall of the pipe, 
concrete pipe with the rubble stacked up against the sides, leaving the open region of the pipe easily accessible. One side of the testbed has two floor slabs staggered such that measurements can be taken through either a single slab or both slabs. The floor slabs were designed and built to represent accurately those that are commonly found in high-rise buildings. They are 6 " thick 3000 psi concrete with $1 / 2$ " diameter rebar on a square lattice with 6 " spacing. The concrete pipe is 4 feet in diameter, and is made in two 3-foot-long sections butted together to create a 6 -foot-long tunnel. The pipe wall is $5^{\prime \prime}$ thick 4000 psi concrete with $1 / 2$ " rebar hoops spaced by 8 ". This meets the ASTM standards for D loading a 6-foot long tube with a force equivalent to the full weight of the two slabs.

Experiments involving human subjects were performed with the prototype RRR at the rubble pile testbed. The subjects were asked to enter the concrete tube, and to sit still. The RRR signals were then acquired during normal breathing, exaggerated breathing and occasionally with the subject moving his hand back and forth slightly. Similar experiments were performed with the RRR in different orientations and through various regions of the rubble pile testbed.

Figure 7 shows an example of the signals recorded through a single floor slab. The photograph in Figure 2a of the experiment clearly shows the rubble pile testbed, the placement of the radar, and the location of the subject. The raw signal as recorded by the RRR is shown in Figure $2 b$ and its Fourier transform (FT) is shown in Figure 2d. Figure $2 c$ is a blow-up of a section of the raw signal, clearly showing the modulation due to the breathing motion of the subject. A very clear peak corresponding to this motion can be seen in FT of the signal at the breathing frequency. The height of this peak compared to the background indicates the cffectiveness of the RRR to detect the presence of the live subject.

\section{Speech Sensors}

A major impediment to speech research is the lack of readily available scientific means of measuring vocal tract motion while speaking. We have conducted several experiments with MIR sensors and have demonstrated that radar measurements of the vocal cords, tongue, and lip motions can be obtained readily. These measurements can possibly provide a direct indication of the vocalized excitation source (i.e. vocal folds opening and closing). In conjunction with the conventional microphone, one could derive the vocal tract transfer function (or voice print) needed for speech synthesis, recognition, compression, and other important applications through the measured motions of the vocal folds, tongue, velum, jaw, and lips. We have made significant progress in the development of MIR based speech sensors relating to how the radar senses the moving object, how the internal filters distort the output of the radar, and how the radar senses movement inside the body.

1. Calibration of the MIR sensor by analyzing its response to sinusoidal vibrations of pistons and injected signals.

Results: A good estimate of the transfer function was obtained. Further experiments were conducted by directly injecting electronic signals into the radar electronics. These more precise measurements allowed to the construction of an inverse digital filter to remove most of the effects of the radar's filters on the signal.

2. Analysis of the radar signals received from rotating Plexiglas disks with embedded holes that simulate the vocal fold movements in the radar field (see Figure 8).

Results: The data analysis revealed much about the way the field disturbance radar operates. The experiment has been invaluable in terms of understanding and explaining how this type of radar operates. In the following graph, the results are plotted. 
and the random rubble plus the wall of the pipe. Breathing and heart beat signals were also recorded of a seated human subject at a distance of 1 meter with no obstructions.

In order to conduct tests of the RRR's performance under conditions similar to those for which it was designed, a mock rubble pile was constructed on site at LLNL. This rubble pile testbed was designed so that instruments and/or human subjects could safely and easily be placed behind both concrete floor slabs as well as randomly distributed concrete, asphalt and rebar debris. A Photograph of the rubble pile testbed is included in Figure 7. The testbed consists of a
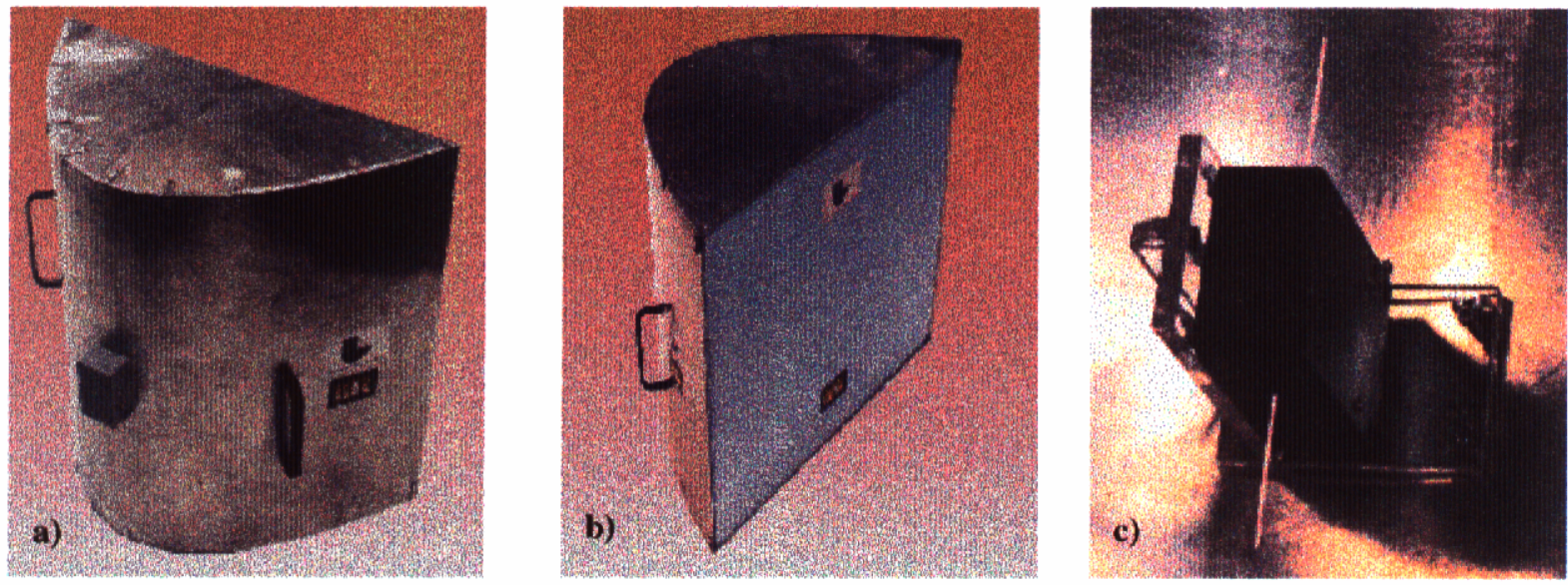

Figure 6 Photographs of the RRR. (a) and (b) two views of the RRR, (c) close up of the MIR transmitter/receiver (T/R). The T/R is positioned with its antenna along the line focus of the
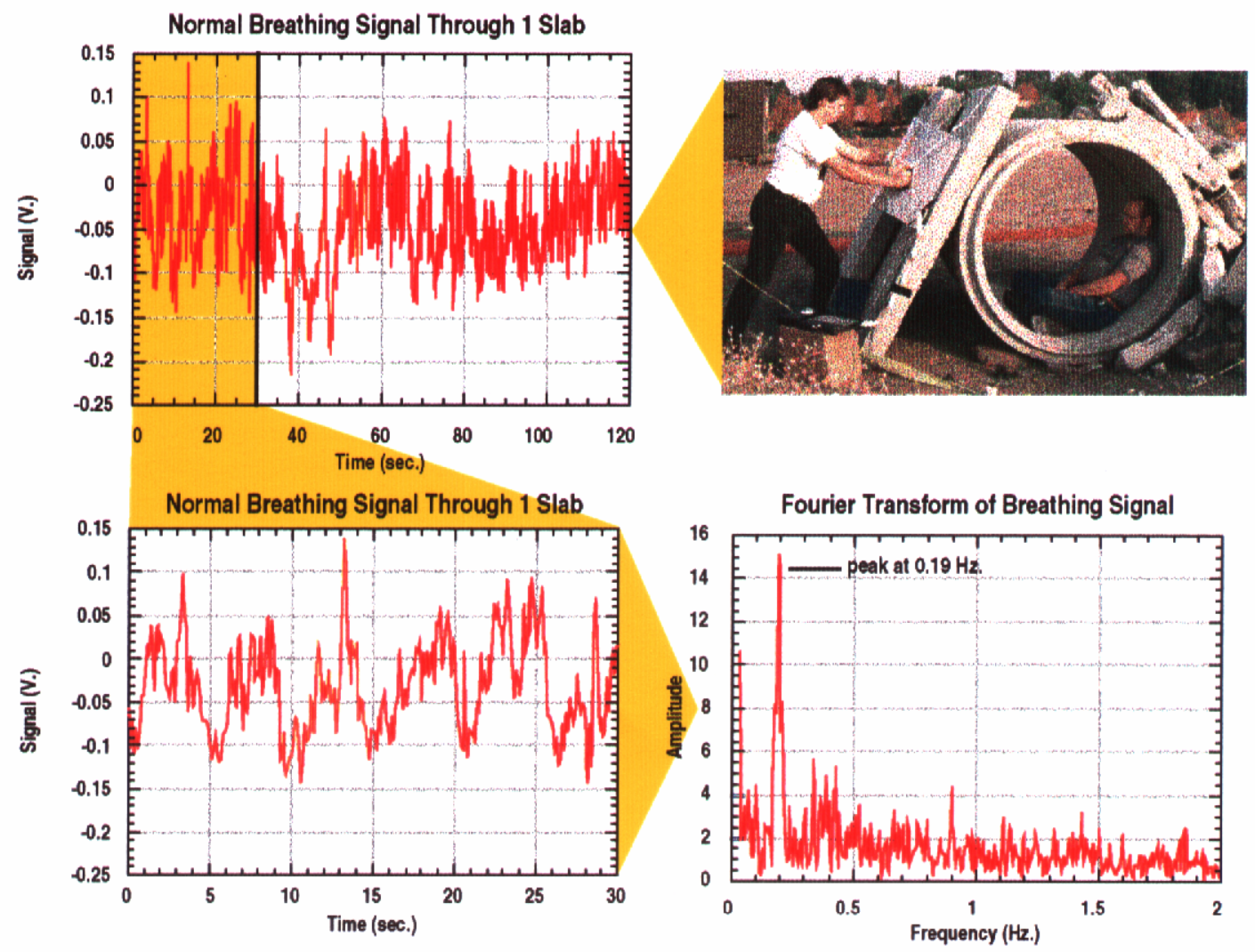

Figure 7 Breathing signals of a human subject recorded with the RRR through a single reinforced concrete floor slab and a concrete pipe. a: a photograph showing breathing detection experiments at the rubble pile testbed located on site at LLNL. b: the raw signal as recorded by the RRR. c: expanded plot of the highlighted region in $b$. The breathing motion is clearly visible as a large modulation in the signal with a period of about 5 seconds. d: the Fourrier transform of the raw signal showing a prominent peak at the breathing frequency. 

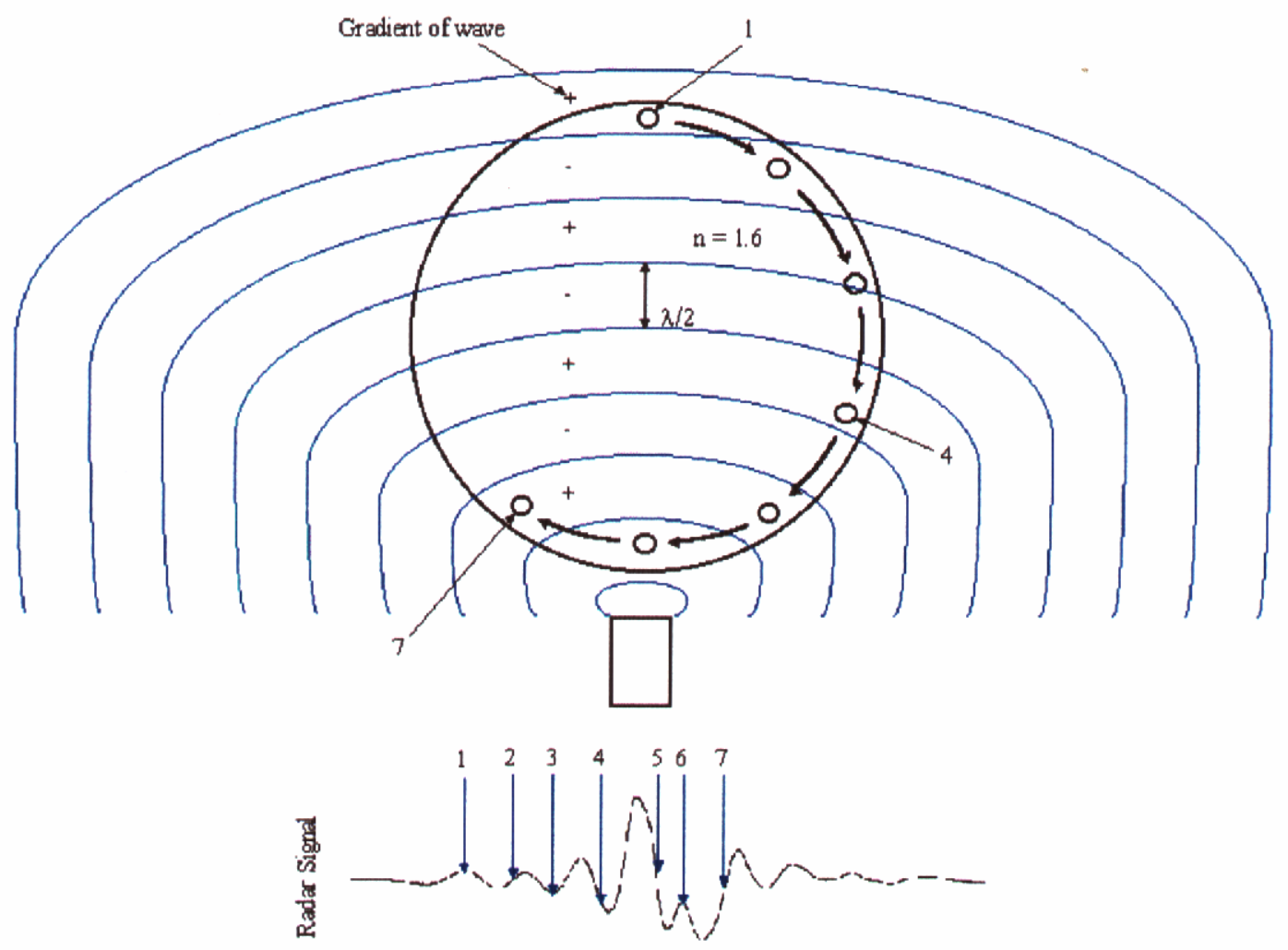

Figure 8 Diagram showing experimental setup for analysis of radar signals received from rotating Plexiglas disks with embedded hols that simulate the vocal fold movements in the radar field.

The disk has a single hole in it with the inside covered by highly reflective copper tape. The series of holes is in time the hole starts at position 1 and goes to position 7 in a clockwise manner. The radar, at the bottom of the disk, emits pulses that travel up through the Plexiglas disk. The waves are bent into the Plexiglas due to the higher dielectric constant of the disk. However, as the waves are traveling at the speed of light and are emitted 2 million times a second, the slowly turning (a few hundred rpms) disk "feels" as if it is traveling trough a stationary wave. The hole passes through several wavelengths of the "frozen" radar wave on its way around the disk. The blue lines represent the radar wave's peaks and valleys, the places where the gradient of the wave changes. Every time the hole traverses on of these peaks, the emitted radar signal changes gradient and the reflected signal changes sign. The signal corresponding to different hole positions is shown under the disk and radar.

What we get from this is a good understanding of how the radar senses movement and how we can take advantage of this method. To get a large signal from the radar you can either have a large motion anywhere or a small movement near a portion of the signal that is changing rapidly. If you are halfway between a peak and trough (at a zero crossing), the radar signal gradient is strongest. It is also most linear. Therefore, for the least amount of distortion it is necessary to place the moving object as near to the zero crossing of the radar wave as possible.

Conversely, if the signal looks distorted or is inverted, it may be that the reflection is coming from the area of one of the peaks or troughs. Understanding this has assisted in the interpretation of the reflected wave from complicated body tissues enormously.

3. Studies including video and airflow data that demonstrate MIR radar provides better cues to pitch, intensity, and linguistic events (glottal stop and vocal fry) than acoustic measurements can provide (see Figure 9).

The results from these experiments (both from the University of Iowa's National Center for Voice and Speech and the UC Davis Voice/Speech/Swallowing Center) have been somewhat surprising - all of the significant radar return appears to come from the front wall of the trachea, which vibrates in concert with the modulation of airflow by the folds. That is, while the relationship between the operation of the folds and the radar return is very strong, it is an 
indirect one. The folds modulate airflow through the glottis, which causes the surrounding tissues to swell and contract as airflow is constrained and then released. The subglottal tissue is very flat, has a large reflective area, and vibrates with an amplitude large enough for the radar to detect (on the order of 100 micrometers, from our experiments with the vibrating pistons). Further analysis is underway to confirm these findings and a model is being produced so that tracheal motion may be related to subglottal pressure and then airflow through the glottis. Some pictures of the experiments at UC Davis are enclosed. They show the use of a laryngoscope (both oral and nasal) which was used to record data into a Kodak EktaPro digital camera. The 3000-fps video was then compared to simultaneous audio, radar, and EGG (electroglottograph) data to ascertain the source of the reflections of the signals.

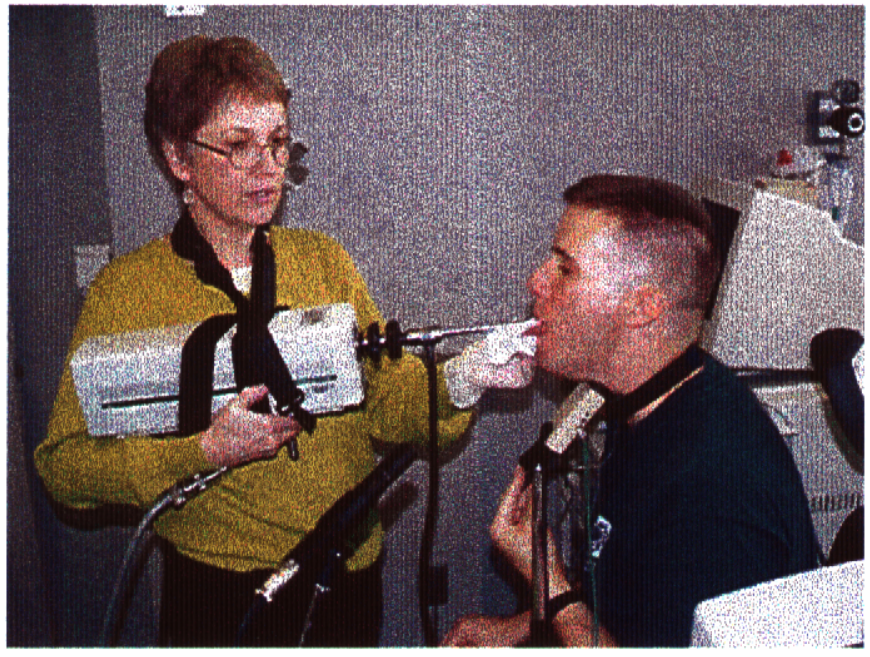

Figure 9 Gathering data with a laryngoscope coupled to a high speed digital movie camera, an EGG, the MIR speech sensor, and a microphone at the UC Davis medical center.

Good progress has also been made in the speech synthesis and speaker verification areas. The speech synthesis modeling has improved dramatically in the last six months and we are now able to replicate individual speakers quite well at single frequencies. In the next fiscal year we will improve this synthesis to include natural sounding frequency variations. The speaker verification project has been aided by the acquisition of a large corpus of data to aid in the verification and synthesis experiments. Over 600 individual sounds and 108 sentences ( 15 repetitions of each) were digitally recorded from 15 male subjects between 24 - 62 for a total of 10,620 structures. The data will be used to test how stable a subject's voice remains over a period of weeks and months as well as what algorithms work best for verification. With the improvements due to the inverse filtering of the radar and results from the video experiments, we hope to build a simple speaker verification system that will demonstrate the power and convenience of utilizing the radar to solve an ongoing speech sciences problem.

A collaboration with the University of California at Berkeley has been initiated with the transfer of several data sets to speech scientists looking at improving conventional speech recognizers by using radar data that we can supply. By using the radar to supplement the audio stream, they hope to improve the accuracy of an HMM recognizer, demonstrating a potential use of the radar in speech science.

\section{Summary}

The LLNL-developed MIR technology has become the foundation for the development of more elaborate systems, such as imaging systems, where multiple sensors have been coupled with advanced signal processing techniques. Over the past several years we have concentrated on a number of applications of MIR which address several remote-sensing applications relevant to emerging programs in defense, transportation, medical, and environmental research. We have successfully established WFO reimbursables in many application areas. Although the research focus has been in the areas of underground imaging, speech technologies, medical signal processing, and personnel detection, we have successfully secured funding in other areas as well. A DOD/NAWC project to monitor the helicopter blades of the V22 Osprey was started in May 1997. The MIR wall surveyor project for OST has been a continuing effort. Also, we have successfully transitioned programs in underground imaging to external funding sources such as the FHWA bridge inspection project. A Defense Special Weapons Agency Project to develop a portable hand-held MIR mine detection 
technology was started in May 1996. A DOE funded project) for the development of security sensors to employ with the ARGUS systems was started in FY97.

\section{Acknowledgments}

The authors would like to acknowledge the contributions of the scientists, engineers and technicians involved with this project. These include J. Burke, G. Dallum, G. Freeze, D. Gavel, H. Jones, S. Nelson, T. Rosenbury, R. Stever, T. Story, J. Trebes, and M. Vigars, P. Welsh.

The authors would like to thank Michael A. Dillabough of the Army Corps of Engineers Earthquake Preparedness Center of Expertise in San Francisco for funding the initial phases of this work and technical information on search-and-rescue techniques and problems. The author also thanks Vince Bormolini of Western Concrete for providing the concrete pipes, and for information on the ASTM codes.

For assistance with the tissue dielectric measurements, thanks go to Mr. Murray, Ms. Grenda Fagin surgical attendants at WRAIR, James Bursani and Hewlell Packard for providing the HP8720-C network analyzer.

\section{Publications}

[1] S. G. Azcvcdo and T. E. McEwan, "Micropower Impulse Radar," Science and Technology Review, UCRL-52000-96-1/2, January 1996.

[2] Popular Science cover story, March 1995. New Scientist cover story, August 1995.

[3] D. T. Gavel, J. E. Mast, J. Warhus, S. G. Azevedo, "An Impulse Radar Array for Detecting Land Mines," Proceedings of the Autonomous Vehicles in Mine Countermeasures Symposium, Monterey CA, p 6-112-120, 4-7 April 1995.

[4] S. G. Azevedo, D.T. Gavel, J. E. Mast, J.P. Warhus, "Landmine Detection and Imaging using Micropower Impulse Radar (MIR)," Proceedings of the Workshop on Anti-personnel Mine Detection and Removal, Lausanne, Switzerland, p48-51, 1 July 1995.

[5] J. E. Mast and E .M. Johansson, "Three-dimensional ground penetrating radar imaging using multi-frequency diffraction tomography," SPIE Vol. 2275, Advanced Microwave and Millimeter Wave Detectors, p 25-26, July 1994.

[6] E. M. Johansson and J.E. Mast, "Three-dimensional ground penetrating radar imaging using synthetic aperture timedomain focusing", SPIE Vol. 2275, Advanced Microwave and Millimeter Wave Detectors, July 1994.

[7] J. E. Mast and S. G. Azevedo, "Applications of Micropower Impulse Radar to Nondestructive Evaluation," Engineering Research, Development and Technology, FY95 Thrust Area Report, Lawrence Livermore National Laboratory, Livermore, Calif. (UCRL 53868-93)

[8] G. C. Burnett, L. C. Ng, W. A. Lee, "Speech articulator measurements using low power EM-wave sensors", J. Acoust. Soc. Am. 103 (1), January 1998.

[9] Anderson, C., W. Aimonetti, M. Barth, M. Buhl, N. Bull, M. Carter, G. Clark, D. Fields, S. Fulkerson, R. Kane, C. Lee, F. Lee, B. McKinley, J. Page, F. Roeske, Jr., T. Rossow, P. Sargis, J. Scarafiotti, P. Schaich, S. Sengupta, R. Sherwood, (1994), "LLNL Electro-optical Mine Detection Program," Lawrence Livermore National Laboratory, Calif. (UCRL-ID118672).

[10] Azevedo, S. G., D. T. Gavel, J. E. Mast, J. P. Warhus, (1995), "Statement of Capabilities: Micropower Impulse Radar (MIR) Technology Applied to Mine Detection and Imaging," Lawrence Livermore National Laboratory, Calif. (UCRLID-5366).

[11] Azevedo, S. G., J. E. Mast, S. D. Nelson, E. T. Rosenbury, H. E. Jones,T. E. McEwan, D. J. Mullenhoff, R. E. Hugenberger, R. D. Stever, J. P. Warhus, M. G. Wieting (1996), "HERMES: A high-speed radar imaging system for inspection of bridge decks," Nundestructive Evaluation Techiques for Aging Infrastructure and Manufacturing, SPIE Vol. 2946, p 195-204.

[12] Azevedo, S. G., T. E. McEwan, and J. P. Warhus (1996), "Microradar Development," Engineering Research, Development and Technology: Thrust Area Report, Lawrence Livermore National Laboratory, Livermore, Calif. (UCRL53868-95), p 6-17.

[13] Azevedo, S. G., D. T. Gavel, J. E. Mast, E. T. Rosenbury, and J. P. Warhus (1996), "Arrays of Micropower Impulse Radar (MIR) scrisors for Subsurfacc Detection," Proceedings of the EURLL Conference on the Detection of Abandoned Land mines, IEE Conf. Pub. No. 431, Edinburgh, Scotland, UK. 
[14] Azevedo, S. G., and T. E. McEwan (1996), "Micropower Impulse Radar," Science and Technology Review, Lawrence Livermore National Laboratory, Livermore, Calif. (UCRL-52000-96-1/2), p 16-29.

[15] Azevedo, S. G., D. T. Gavel, J. E. Mast, J. P. Warhus (1995), "Landmine Detection and Imaging using Micropower Impulse Radar (MIR)," Proceedings of the Workshop on Anti-personnel Mine Detection and Removal, Lausanne, Switzcrland, $\mathrm{p} 48-51$.

[16] Clark, G. A., J. E. Hernandez, S. K. Sengupta, R. J. Sherwood, P. C. Schaich, M. R. Buhl, R. J. Kane, M. J. Barth, N. K. DelGrande (1992), "Computer Visioin and Sensor Fusion for Detecting Buried Objects," Proceedings of the 26th Asilomar Conference on Signals, Systems \& Computers, Pacific Grove, Calif., IEEE, p 466.

[17] Clark, G. A., S. K. Sengupta, W. D. Aimonetti, F. Roeske, J. G. Donetti, D. J. Fields, R. J. Sherwood, P. C. Schaich (1995), "Computer Visioin and Sensor Fusion for Detecting Buried Objects," Proceedings of the Symposium of Autonomous Systems in Mine Countermeasures, Monterey, Calif, April 4-7, 1995.

[18] Gavel, D. T., J. E. Mast, J. Warhus, and S. G. Azevedo (1995), "An Impulse Radar Array for Detecting Landmines," Proceedings of the Autonomous Vehicles in Mine Countermeasures Symposium, Monterey, Calif, p 6-112.

[19] Popular Science (March 1995), Radar on a Chip: 101 Uses in your Life.

[20] New Scientist (August 1995), The Pocket Radar Revolution.

[21] Nelson, S. D. (1994), "Electromagnetic Modeling for Target-Rich Embedded Environments," Engineering Research, Development, and Technology, Lawrence Livermore National Laboratory, Livermore, California, (UCRL-53868-93).

[22] Sargis, P. D., F.D. Lee, E.S. Fulkerson, B.J. McKinley, W.D. Aimonetti (1994), "Ground-Penetrating Radar for Buried Mine Detection," Aerial Surveillance Sensing, including Obscured and Underground Object Detection, SPIE Vol. 2217, 4-6 April 1994.

[23] Warhus, J. P., J. E. Mast, E. M. Johansson, S. D. Nelson (1994), "Advanced ground penetrating radar," Advanced Microwave and Millimeter Wave Detectors, SPIE Vol. 2275, p 22-24. 


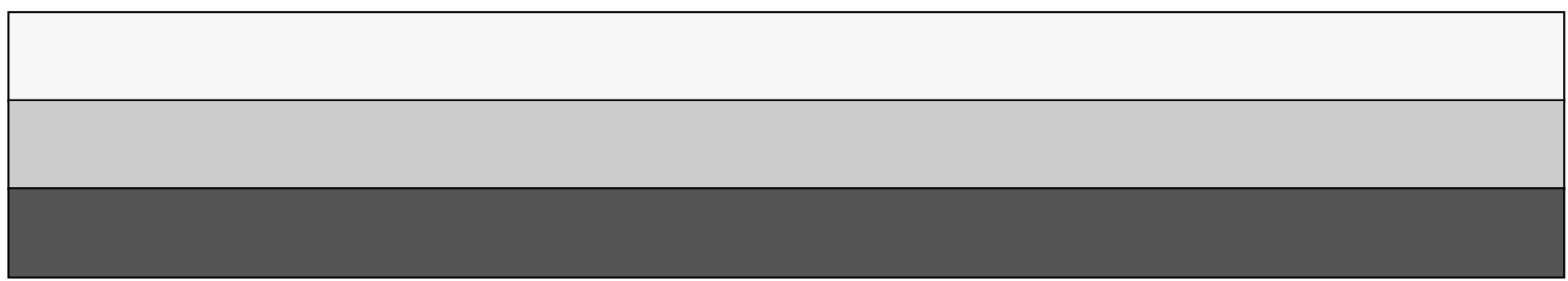

